\title{
Single integrodifferential wave equation for a Lévy walk
}

DOI:

10.1103/PhysRevE.93.020101

\section{Document Version}

Final published version

Link to publication record in Manchester Research Explorer

\section{Citation for published version (APA):}

Fedotov, S. (2016). Single integrodifferential wave equation for a Lévy walk. Physical review. E, Statistical, nonlinear, and soft matter physics, 93, [020101 (R)]. https://doi.org/10.1103/PhysRevE.93.020101

\section{Published in:}

Physical review. E, Statistical, nonlinear, and soft matter physics

\section{Citing this paper}

Please note that where the full-text provided on Manchester Research Explorer is the Author Accepted Manuscript or Proof version this may differ from the final Published version. If citing, it is advised that you check and use the publisher's definitive version.

\section{General rights}

Copyright and moral rights for the publications made accessible in the Research Explorer are retained by the authors and/or other copyright owners and it is a condition of accessing publications that users recognise and abide by the legal requirements associated with these rights.

\section{Takedown policy}

If you believe that this document breaches copyright please refer to the University of Manchester's Takedown Procedures [http://man.ac.uk/04Y6Bo] or contact uml.scholarlycommunications@manchester.ac.uk providing relevant details, so we can investigate your claim.

\section{OPEN ACCESS}




\title{
Single integrodifferential wave equation for a Lévy walk
}

\author{
Sergei Fedotov \\ School of Mathematics, The University of Manchester, Manchester M13 9PL, United Kingdom
}

(Received 18 September 2015; published 1 February 2016)

\begin{abstract}
We derive the single integrodifferential wave equation for the probability density function of the position of a classical one-dimensional Lévy walk with continuous sample paths. This equation involves a classical wave operator together with memory integrals describing the spatiotemporal coupling of the Lévy walk. It is valid at all times, not only in the long time limit, and it does not involve any large-scale approximations. It generalizes the well-known telegraph or Cattaneo equation for the persistent random walk with the exponential switching time distribution. Several non-Markovian cases are considered when the particle's velocity alternates at the gamma and power-law distributed random times. In the strong anomalous case we obtain the asymptotic solution to the integrodifferential wave equation. We implement the nonlinear reaction term of Kolmogorov-PetrovskyPiskounov type into our equation and develop the theory of wave propagation in reaction-transport systems involving Lévy diffusion.
\end{abstract}

DOI: 10.1103/PhysRevE.93.020101

Introduction. Lévy walk is a fundamental notion in physics and biology [1] with numerous applications including the transport of light governed by Lévy statistics [2], anomalous superdiffusion of cold atoms in optical lattices [3], T-cell motility in the brain [4], endosomal active transport within living cells [5]. In the last few years, the interest in the Lévy walk models increases rapidly in the context of Lévy flight foraging hypothesis according to which a Lévy transport is optimal to search for randomly located objects [6]. The Lévy movement pattern has been observed in microorganisms, insects, molluscs, birds, etc. [7]. A recent review [8] provides a detailed discussion of existing applications and the most current status of Lévy walk theory.

The standard model for a Lévy walk is based on the continuous time random walk (CTRW) with coupled probability density function for the jump length and the waiting time between two successive jumps. Many contributions on this subject have been given since the pioneering works three decades ago (see, for example, [9]). Two integral equations for the probability density function (PDF) $p(x, t)$ for a walker position $x$ and arrival rate $j(x, t)$ can be formulated and solved $[1,8]$. An equivalent single integral equation for $p(x, t)$ has been also formulated $[10,11]$. To describe a Lévy walk spatiotemporal coupling, two dynamical equations have been suggested in [12]. Another approach to describe the superdiffusive behavior is based on the analysis of joint PDF $p(x, v, t)$ of the particle's position $x$ and velocity $v$. Various fractional generalizations of Kramers-Fokker-Planck equation for $p(x, v, t)$ have been derived [13-15].

It is well known that the alternative formulation of the uncoupled CTRW with the independent random jump lengths and waiting times is the generalized master equation for the PDF $p(x, t)$ with memory kernel $K(\tau)$ [16]. The main result of this Rapid Communication is to provide an analogous equation for the Lévy walk PDF $p(x, t)$. We obtain the following integrodifferential wave equation:

$$
\begin{aligned}
& \frac{\partial^{2} p}{\partial t^{2}}-v^{2} \frac{\partial^{2} p}{\partial x^{2}}+\int_{0}^{t} \int_{V} K(\tau) \varphi(u)\left(\frac{\partial}{\partial t}-u \frac{\partial}{\partial x}\right) \\
& \quad \times p(x-u \tau, t-\tau) d u d \tau=0
\end{aligned}
$$

where $v$ is a constant speed of walker, $\varphi(u)$ is the velocity jump density,

$$
\varphi(u)=\frac{1}{2} \delta(u-v)+\frac{1}{2} \delta(u+v),
$$

in the velocity space $V$. The standard memory kernel $K(\tau)$ is determined by its Laplace transform $\hat{K}(s)=\hat{\psi}(s) / \hat{\Psi}(s)$, where $\hat{\psi}(s)$ and $\hat{\Psi}(s)$ are the Laplace transforms of the running time density $\psi(\tau)$ and the survival function $\Psi(\tau)$ [1]. From Eq. (1) with $p(x, 0)=p_{0}(x)$ and $p_{t}(x, 0)=0$, one can obtain the well-known expression for the Fourier-Laplace transform of the PDF $p(x, t)$,

$$
\hat{p}(k, s)=\frac{[\hat{\Psi}(s+i k v)+\hat{\Psi}(s-i k v)] \hat{p}_{0}(k)}{2-\hat{\psi}(s+i k v)-\hat{\psi}(s-i k v)},
$$

where $\hat{p}_{0}(k)=\int_{\mathbb{R}} p_{0}(x) e^{i k x} d x[8]$.

Derivation of single equation. We consider the Lévy walk as the random particle's motion with continuous sample paths (no jumps) along one-dimensional space. The particle starts to move with constant speed $v$ at time $t=0$ and after a random time (running time) it either continues the movement in the same direction with curtain probability or changes the direction and moves with the same constant speed. The random running time is defined by the switching rate $\gamma(\tau)$ or the running time $\operatorname{PDF} \psi(\tau)=\gamma(\tau) \exp \left[-\int_{0}^{\tau} \gamma(s) d s\right]$. To derive the governing equation for the Lévy walk, we start with the Markovian model involving structural densities with the extra running time variable $\tau$ [17-21]. We define the structural PDF's of walker, $n_{+}(x, t, \tau)$, at point $x$ and time $t$ that moves in the right direction, $(+)$, with constant speed $v$ during time $\tau$ since the last switching. The probability density function $n_{-}(x, t, \tau)$ corresponds to the walker that moves in the negative direction, (-). The balance equations for both structural PDF's $n_{+}(x, t, \tau)$ and $n_{-}(x, t, \tau)$ can be written as

$$
\frac{\partial n_{ \pm}}{\partial t} \pm v \frac{\partial n_{ \pm}}{\partial x}+\frac{\partial n_{ \pm}}{\partial \tau}=-\gamma(\tau) n_{ \pm},
$$

where the switching rate $\gamma(\tau)$ depends on the running time $\tau$. If the walker moves in the positive direction it can switch with rate $\gamma(\tau)$ either to the opposite direction with the probability $\alpha_{-}$or keep the same direction with the probability $\alpha_{+}$such that 
$\alpha_{+}+\alpha_{-}=1$. For the walker moving in the negative direction corresponding characteristics are $\beta_{+}$and $\beta_{-}$. The well-known velocity model and two-state model are just particular cases of this general two-state model. For example, the choice $\alpha_{+}=\beta_{-}=0$ and $\alpha_{-}=\beta_{+}=1$ corresponds to the two-state model [22]. The probabilities $\alpha_{ \pm}=\beta_{ \pm}=1 / 2$ correspond to the velocity model [23]. We assume that all walkers start to move with zero running time $\tau$,

$$
n_{ \pm}(x, 0, \tau)=\frac{1}{2} p_{0}(x) \delta(\tau) .
$$

The boundary conditions at zero running time are

$$
\begin{aligned}
n_{ \pm}(x, t, 0)= & \alpha_{ \pm} \int_{0}^{t} \gamma(\tau) n_{+}(x, t, \tau) d \tau \\
& +\beta_{ \pm} \int_{0}^{t} \gamma(\tau) n_{-}(x, t, \tau) d \tau .
\end{aligned}
$$

Our aim is to derive the master equations for the probability density functions $p_{+}(x, t)$ and $p_{-}(x, t)$ defined as

$$
p_{ \pm}(x, t)=\int_{0}^{t} n_{ \pm}(x, t, \tau) d \tau .
$$

By differentiating (7) with respect to time $t$ and using the balance equations (4) we obtain

$$
\begin{aligned}
\frac{\partial p_{ \pm}}{\partial t}= & n_{ \pm}(x, t, t) \mp v \int_{0}^{t} \frac{\partial n_{ \pm}}{\partial x} d \tau \\
& -\int_{0}^{t} \frac{\partial n_{ \pm}}{\partial \tau} d \tau-\int_{0}^{t} \gamma(\tau) n_{ \pm}(x, t, \tau) d \tau .
\end{aligned}
$$

These equations can be rewritten as the master equations

$$
\frac{\partial p_{ \pm}}{\partial t} \pm v \frac{\partial p_{ \pm}}{\partial x}=j_{ \pm}(x, t)-i_{ \pm}(x, t),
$$

where the rates of switching $i_{ \pm}(x, t)$ are defined as

$$
i_{ \pm}(x, t)=\int_{0}^{t} \gamma(\tau) n_{ \pm}(x, t, \tau) d \tau
$$

and the arrival rates $j_{ \pm}(x, t)=n_{ \pm}(x, t, 0)$. By using the definitions of the switching and arrival rates above, Eq. (6) can be written in the compact form

$$
j_{ \pm}(x, t)=\alpha_{ \pm} i_{+}(x, t)+\beta_{ \pm} i_{-}(x, t) .
$$

In what follows we consider only the simple case of a symmetric Lévy walk for which $\alpha_{ \pm}=\beta_{ \pm}=1 / 2$. In general, the probabilities $\alpha_{ \pm}$and $\beta_{ \pm}$can be useful to formulate the impact of the external force. Substitution of (10) with $\alpha_{ \pm}=\beta_{ \pm}=1 / 2$ into (8) gives

$$
\begin{gathered}
\frac{\partial p_{+}}{\partial t}+v \frac{\partial p_{+}}{\partial x}=-\frac{1}{2} i_{+}(x, t)+\frac{1}{2} i_{-}(x, t), \\
\frac{\partial p_{-}}{\partial t}-v \frac{\partial p_{-}}{\partial x}=\frac{1}{2} i_{+}(x, t)-\frac{1}{2} i_{-}(x, t),
\end{gathered}
$$

where the switching rates $i_{ \pm}(x, t)$ can be found as follows. By the method of characteristics, we find from (4)

$$
n_{ \pm}(x, t, \tau)=n_{ \pm}(x \mp v \tau, t-\tau, 0) \Psi(\tau), \quad \tau<t,
$$

where $\Psi(\tau)$ is the survival function

$$
\Psi(\tau)=e^{-\int_{0}^{\tau} \gamma(s) d s} .
$$

Note that at $\tau=t$ we have a singularity due to the initial condition (5). Substitution of (13) into (9) and (7) together with the initial condition (5) gives

$$
\begin{aligned}
& i_{ \pm}(x, t)=\int_{0}^{t} j_{ \pm}(x \mp v \tau, t-\tau) \psi(\tau) d \tau+\frac{1}{2} p_{0}(x \mp v t) \psi(t), \\
& p_{ \pm}(x, t)=\int_{0}^{t} j_{ \pm}(x \mp v \tau, t-\tau) \Psi(\tau) d \tau+\frac{1}{2} p_{0}(x \mp v t) \Psi(t) .
\end{aligned}
$$

Applying the Fourier-Laplace transform to these equations, we find expressions for $i_{+}(x, t)$ and $i_{-}(x, t)$ [21]

$$
i_{ \pm}(x, t)=\int_{0}^{t} K(\tau) p_{ \pm}(x \mp v \tau, t-\tau) d \tau .
$$

Note that four equations $i_{ \pm}(x, t)$ and $p_{ \pm}(x, t)$ can be written in the standard form of two equations for

$$
p(x, t)=p_{+}(x, t)+p_{-}(x, t)
$$

and the arrival rate $j=j_{+}+j_{-}$with the jump density $w(z \mid \tau)=\frac{1}{2} \delta(z-v \tau)+\frac{1}{2} \delta(z+v \tau):$

$$
\begin{aligned}
p(x, t)= & \int_{0}^{t} \int_{\mathbb{R}} j(x-z, t-\tau) w(z \mid \tau) \Psi(\tau) d z d \tau \\
& +\int_{\mathbb{R}} p_{0}(x-z) w(z \mid t) d z \Psi(t), \\
j(x, t)= & \int_{0}^{t} \int_{\mathbb{R}} j(x-z, t-\tau) w(z \mid \tau) \psi(\tau) d z d \tau \\
& +\int_{\mathbb{R}} p_{0}(x-z) w(z \mid t) d z \psi(t) .
\end{aligned}
$$

Our main purpose now is to reduce the system (11) and (12) with (15) to a single governing equation for the PDF $p(x, t)$ defined by (16). First we introduce the flux [24],

$$
J(x, t)=v p_{+}(x, t)-v p_{-}(x, t) .
$$

Then by adding (11) and (12) we obtain the standard conservation equation

$$
\frac{\partial p}{\partial t}+\frac{\partial J}{\partial x}=0
$$

Equation for the flux $J$ can be obtained by multiplication of (11) and (12) by $v$ and subtraction,

$$
\frac{\partial J}{\partial t}+v^{2} \frac{\partial p}{\partial x}=-v\left[i_{+}(x, t)-i_{-}(x, t)\right] .
$$

By differentiating (18) with respect to $t$ and (19) with respect to $x$ and eliminating $\partial^{2} J / \partial t \partial x$, we obtain

$$
\frac{\partial^{2} p}{\partial t^{2}}=v^{2} \frac{\partial^{2} p}{\partial x^{2}}+v \frac{\partial}{\partial x}\left[i_{+}(x, t)-i_{-}(x, t)\right] .
$$

Now we need to express the last term in (20) in terms of $p(x, t)$ alone. From (16) and (17) we find the expressions for $p_{+}(x, t)$ and $p_{-}(x, t)$ in terms of the PDF $p(x, t)$ and the flux $J(x, t)$,

$$
p_{ \pm}(x, t)=\frac{p(x, t)}{2} \pm \frac{J(x, t)}{2 v} .
$$


By using (18), (21), and (15) we obtain from (20) a single integrodifferential equation for the $\operatorname{PDF} p(x, t)$,

$$
\begin{aligned}
\frac{\partial^{2} p}{\partial t^{2}}= & v^{2} \frac{\partial^{2} p}{\partial x^{2}} \\
& -\frac{1}{2} \int_{0}^{t} K(\tau)\left[\left(\frac{\partial}{\partial t}-v \frac{\partial}{\partial x}\right) p(x-v \tau, t-\tau)\right] d \tau \\
& -\frac{1}{2} \int_{0}^{t} K(\tau)\left[\left(\frac{\partial}{\partial t}+v \frac{\partial}{\partial x}\right) p(x+v \tau, t-\tau)\right] d \tau .
\end{aligned}
$$

This integrodifferential wave equation for a Lévy walk is valid for any running time PDF $\psi(\tau)$. It generalizes the well-known telegraph equation obtained from the persistent random walk with the constant rate of switching $\gamma$. By using the velocity jump density (2), we rewrite Eq. (22) in the compact form (1). It is instructive to compare the uncoupled CTRW with Lévy walk. When jumps and waiting times of the CTRW are independent random variables, one can convert the single integral equation for $p(x, t)$ into a master equation $[16,17]$. For the Lévy walk involving spatiotemporal coupling the integrodifferential wave equation (22) plays the same role as the master equation for the uncoupled CTRW. Let us now consider several examples of the running time PDF $\psi(\tau)$.

Exponential running time density. In the Markovian case with the exponential running time PDF: $\psi(\tau)=$ $T^{-1} \exp \left(-\tau T^{-1}\right)$ for which $\hat{\psi}(s)=(1+T s)^{-1}$ and $K(\tau)=$ $T^{-1} \delta(\tau)$, we obtain from (22) the classical Cattaneo or telegraph equation [17]

$$
\frac{\partial^{2} p}{\partial t^{2}}+\frac{1}{T} \frac{\partial p}{\partial t}-v^{2} \frac{\partial^{2} p}{\partial x^{2}}=0
$$

This hyperbolic equation ensures that the density profile propagates with finite speed $v$.

Gamma PDF $g(\tau, 2, \lambda)$. For the biological applications it is important to consider a running time PDF that takes the maximum value not at zero time as the exponential density [7]. For example, it was found that the running time density for a single bacterium might deviate significantly from exponential approximation [25]. One example of such PDF is the gamma density:

$$
\psi(\tau)=g(\tau, 2, \lambda)=\lambda^{2} \tau \exp (-\lambda \tau)
$$

with $\hat{\psi}(s)=\lambda^{2}(s+\lambda)^{-2}$ and

$$
\hat{K}(s)=\frac{\lambda^{2}}{2 \lambda+s} .
$$

The memory kernel in Eq. (22) takes the form $K(\tau)=$ $\lambda^{2} \exp (-2 \lambda \tau)$. The advantage of this exponential memory kernel is that one can localize integrodifferential equation (22) by direct differentiation of (22) with respect to time twice:

$$
\begin{aligned}
& \frac{\partial^{4} p}{\partial t^{4}}+4 \lambda \frac{\partial^{3} p}{\partial t^{3}}+5 \lambda^{2} \frac{\partial^{2} p}{\partial t^{2}}+2 \lambda^{3} \frac{\partial p}{\partial t} \\
& =v^{2} \frac{\partial^{2}}{\partial x^{2}}\left[2 \frac{\partial^{2} p}{\partial t^{2}}+4 \lambda \frac{\partial p}{\partial t}+3 \lambda^{2} p-v^{2} \frac{\partial^{2} p}{\partial x^{2}}\right] .
\end{aligned}
$$

Note that non-Markovian particle's movement with velocities alternating at Erlang-distributed and gamma-distributed random times was considered in [26]. Next we consider the anomalous case involving walker's velocities alternating at power-law distributed random times [12,20-22].

Anomalous enhanced transport. We consider two anomalous cases: (1) strong ballistic case for which the mean squared displacement, $\left\langle x^{2}\right\rangle \sim t^{2}$, and (2) subballistic superdiffusion with $\left\langle x^{2}\right\rangle \sim t^{3-\mu}$, where $1<\mu<2$ [8].

In the strong ballistic case, we use the survival function

$$
\Psi(\tau)=E\left[-\left(\tau / \tau_{0}\right)^{\mu}\right], \quad 0<\mu<1,
$$

where $E[z]$ is the Mittag-Leffler function, $\tau_{0}$ is a constant with the unit of time. In this case the mean running time is divergent $[27,28]$, the Laplace transform of the running time PDF $\psi(\tau)$ is

$$
\hat{\psi}(s)=\frac{1}{1+\left(\tau_{0} s\right)^{\mu}}, \quad 0<\mu<1,
$$

and the Laplace transform of the memory kernel $K(\tau)$ is

$$
\hat{K}(s)=\frac{s \hat{\psi}(s)}{1-\hat{\psi}(s)}=\frac{s^{1-\mu}}{\tau_{0}^{\mu}} .
$$

It is important to note that this memory kernel is valid at all times and it does not involve the standard long-time asymptotic $s \rightarrow 0$. The main equation (1) can be rewritten in the different forms involving material fractional derivatives [12,29-33]. We write it in the form

$$
\frac{\partial^{2} p}{\partial t^{2}}-v^{2} \frac{\partial^{2} p}{\partial x^{2}}+\frac{1}{\tau_{0}^{\mu}} \int_{V} \varphi(u) L_{u}^{1-\mu} p d u=0,
$$

where the operator $L_{u}^{1-\mu}$ for $0<\mu<1$ is defined by its Fourier-Laplace transform

$$
\mathcal{F} \mathcal{L}\left\{L_{u}^{1-\mu} p\right\}=(s-i k u)^{1-\mu}\left[(s+i k u) \hat{p}(k, s)-\hat{p}_{0}(k)\right] .
$$

In the subballistic superdiffusive case, one can obtain for small $s$

$$
\hat{\psi}(s) \simeq 1-T s+A T s^{\mu}, \quad 1<\mu<2,
$$

for which the first moment $T=\int_{0}^{\infty} \Psi(\tau) d \tau$ is finite and the second moment is divergent; $A=\Gamma(2-\mu) \tau_{0}^{\mu-1}$. Then the Laplace transform of $K(\tau)$ is $\hat{K}(s) \simeq T^{-1}\left(1+A s^{\mu-1}\right)$ as $s \rightarrow$ 0 . From (1) we obtain

$$
T \frac{\partial^{2} p}{\partial t^{2}}+\frac{\partial p}{\partial t}-D \frac{\partial^{2} p}{\partial x^{2}}+A \int_{V} \varphi(u) L_{u}^{\mu-1} p d u=0,
$$

where the operator $L_{u}^{\mu-1}$ for $1<\mu<2$ is defined by (31), where $1-\mu$ is replaced by $\mu-1$, and the diffusion coefficient $D=T v^{2}$. It is clear from (30) and (33) that strong ballistic and subballistic cases are fundamentally different. In the strong ballistic case $(0<\mu<1)$, the integral term is in the balance with classical wave equation, while for the subballistic superdiffusive case $(1<\mu<2)$, the memory term is in the balance with the Cattaneo (telegraph) equation. One can perform various asymptotic analysis of (30) and (33), obtain the pseudodifferential equations for the walker's PDF position [30-33] and determine the shape of PDF profiles [34].

As an illustration let us find the long-time asymptotic solution to Eq. (30) $(0<\mu<1)$. In the limit $\tau_{0} \rightarrow 0$, the 
evolution of the PDF $p(x, t)$ is determined by the integral term, while the first two waves' terms in (30) are irrelevant. The PDF $p(x, t)$ obeys

$$
\int_{V} \varphi(u) L_{u}^{1-\mu} p d u=0 .
$$

By using (31), we obtain

$$
\begin{aligned}
& (s-i k v)^{1-\mu}\left[(s+i k v) \hat{p}(k, s)-\hat{p}_{0}(k)\right] \\
& \quad+(s+i k v)^{1-\mu}\left[(s-i k v) \hat{p}(k, s)-\hat{p}_{0}(k)\right]=0 .
\end{aligned}
$$

In particular, for $\mu=1 / 2$ and $\hat{p}_{0}(k)=1$, we factorize Eq. (35) and find that

$$
\left(s^{2}+k^{2} v^{2}\right)^{1 / 2} \hat{p}(k, s)-1=0 .
$$

By using the inverse Fourier-Laplace transform we obtain the well-known self-similar profile [1],

$$
p(x, t)=\pi^{-1}\left(v^{2} t^{2}-x^{2}\right)^{-1 / 2} .
$$

For the arbitrary $\mu$ from the interval $0<\mu<1$, one can find from (35) that the solution to (34) is the Lamperti distribution [8]. Note that at the small times $t \ll \tau_{0}$, it follows from (30) that the integral term is irrelevant, so the $p(x, t)$ obeys the wave equation. For the initial conditions $p(x, 0)=\delta(x)$ and $p_{t}(x, 0)=0$ it has the classical d'Alembert's solution $p(x, t)=\frac{1}{2} \delta(x-v t)+\frac{1}{2} \delta(x+v t)$ representing two waves traveling in opposite directions. These two waves are the walkers that never change their directions. Later these $\delta$ functional "horns" develop decaying weight proportional to the power law survival function $t^{-\mu}$ [1]. The shape of the PDF at several successive times can be found in [8]. It would be interesting to use a new wave equation for the nonnormalizable density problem for superdiffusive anomalous transport [35].

Front propagation. Our technique leads to the significant advances in the extension of the standard linear Lévy walk models. The main advantage of our approach is that it allows us to implement the nonlinear reactions and develop the theory of wave propagation [17]. As an illustration let us consider the fundamental problem of wave propagation in reaction-transport systems involving enhanced Lévy diffusion. Instead of the PDF's $n_{ \pm}, p_{ \pm}$, and $p$ we consider the mean densities of Lévy walkers $n_{ \pm}$and $\rho_{ \pm}$. To take into account the nonlinear reaction (proliferation of Lévy walkers), we modify the boundary condition at zero running time as follows:

$$
\begin{aligned}
n_{ \pm}(x, t, 0)= & \frac{1}{2} \int_{0}^{t} \gamma(\tau) n_{+}(x, t, \tau) d \tau+\frac{1}{2} \int_{0}^{t} \gamma(\tau) n_{-}(x, t, \tau) d \tau \\
& +\frac{1}{2} r(\rho) \rho(x, t),
\end{aligned}
$$

where $r(\rho)$ is the density dependent proliferation rate. This boundary condition corresponds to the case when newborn walkers have zero running time. We consider the reaction term of Kolmogorov-Petrovsky-Piskounov (KPP) type [7] for which

$$
\max _{\rho} r(\rho)=r(0)=r, \quad r(1)=0 .
$$

For example, $r(\rho)=r(1-\rho)$ corresponds to the logistic growth. The master equations for the mean densities $\rho_{ \pm}$take the form

$$
\begin{gathered}
\frac{\partial \rho_{+}}{\partial t}+v \frac{\partial \rho_{+}}{\partial x}=-\frac{1}{2} i_{+}(x, t)+\frac{1}{2} i_{-}(x, t)+\frac{r(\rho) \rho}{2} \\
\frac{\partial \rho_{-}}{\partial t}-v \frac{\partial \rho_{-}}{\partial x}=\frac{1}{2} i_{+}(x, t)-\frac{1}{2} i_{-}(x, t)+\frac{r(\rho) \rho}{2} .
\end{gathered}
$$

The governing equation for the mean density $\rho=\rho_{+}+\rho_{-}$is

$$
\frac{\partial \rho}{\partial t}+\frac{\partial J}{\partial x}=r(\rho) \rho,
$$

where the flux $J=v \rho_{+}-v \rho_{-}$obeys the equation

$$
\frac{\partial J}{\partial t}+v^{2} \frac{\partial \rho}{\partial x}=-v\left[i_{+}(x, t)-i_{-}(x, t)\right] .
$$

By differentiating (41) with respect to $t$ and (42) with respect to $x$ and eliminating $\partial^{2} J / \partial t \partial x$, we obtain a single nonlinear integrodifferential equation for the density $\rho(x, t)$,

$$
\begin{aligned}
\frac{\partial^{2} \rho}{\partial t^{2}}-v^{2} \frac{\partial^{2} \rho}{\partial x^{2}}= & {\left[r(\rho)+r^{\prime}(\rho) \rho\right] \frac{\partial \rho}{\partial t}-\int_{0}^{t} \int_{V} K(\tau) \varphi(u) } \\
& \times\left(\frac{\partial}{\partial t}-u \frac{\partial}{\partial x}-r(\rho)\right) \rho d u d \tau
\end{aligned}
$$

where the velocity jump density $\varphi(u)$ is defined by (2). This equation is a generalization of the well-known reactiontelegraph equation [7]. Our purpose now is to find a traveling wave solution to this integrodifferential wave equation with appropriate initial conditions. To ensure the minimal propagation speed we consider the frontlike initial condition

$$
\rho(x, 0)=\left\{\begin{array}{ll}
0, & x \geqslant 0 \\
1, & x<0
\end{array} .\right.
$$

The main idea is that after the hyperbolic scaling $t \rightarrow t / \varepsilon$, $x \rightarrow x / \varepsilon$, the rescaled density $\rho^{\varepsilon}(x, t)=\rho^{\varepsilon}(x / \varepsilon, t / \varepsilon)$ with the KPP-kinetics (38) takes only two values 0 and 1 as $\varepsilon \rightarrow 0$. The rescaled linear equation takes the form

$$
\begin{aligned}
\varepsilon^{2} \frac{\partial^{2} \rho^{\varepsilon}}{\partial t^{2}}-\varepsilon^{2} v^{2} \frac{\partial^{2} \rho^{\varepsilon}}{\partial x^{2}}-r \varepsilon \frac{\partial \rho^{\varepsilon}}{\partial t} \\
=-\frac{\varepsilon}{2} \int_{0}^{t / \varepsilon} K(\tau)\left[\left(\frac{\partial}{\partial t}-v \frac{\partial}{\partial x}-r\right) \rho^{\varepsilon}\right. \\
\left.\quad \times\left(\frac{x}{\varepsilon}-v \tau, \frac{t}{\varepsilon}-\tau\right)\right] d \tau \\
\quad-\frac{\varepsilon}{2} \int_{0}^{t / \varepsilon} K(\tau)\left[\left(\frac{\partial}{\partial t}+v \frac{\partial}{\partial x}-r\right) \rho^{\varepsilon}\right. \\
\left.\quad \times\left(\frac{x}{\varepsilon}+v \tau, \frac{t}{\varepsilon}-\tau\right)\right] d \tau .
\end{aligned}
$$

It is well known that for the reaction-transport systems with the KPP kinetics the front speed is determined by the tail density distribution. Therefore it is enough to consider the linear reaction. We apply an exponential transformation

$$
\rho^{\varepsilon}(x, t)=\exp \left(-\frac{G(x, t)}{\varepsilon}\right)
$$


and obtain the generalized Hamilton-Jacobi equation for $G(x, t)$ as $\varepsilon \rightarrow 0$,

$$
\begin{aligned}
\left(\frac{\partial G}{\partial t}\right)^{2}-v^{2}\left(\frac{\partial G}{\partial x}\right)^{2}+r \frac{\partial G}{\partial t} & 1 \\
= & \frac{1}{2}\left(\frac{\partial G}{\partial t}-v \frac{\partial G}{\partial x}+r\right) \hat{K}\left(-\frac{\partial G}{\partial t}-v \frac{\partial G}{\partial x}\right) \\
& +\frac{1}{2}\left(\frac{\partial G}{\partial t}+v \frac{\partial G}{\partial x}+r\right) \hat{K}\left(-\frac{\partial G}{\partial t}+v \frac{\partial G}{\partial x}\right) .
\end{aligned}
$$

Here $\hat{K}(s)$ is the Laplace transform of the memory kernel $K(\tau)$. The crucial advantage of (46) is that the expression for $\hat{K}(s)$ is always available compared to $K(\tau)$ which is not. For $K(\tau)=\frac{1}{T} \delta(\tau)$ we obtain the relativistic Hamilton-Jacobi equation

$$
\left(\frac{\partial G}{\partial t}\right)^{2}-v^{2}\left(\frac{\partial G}{\partial x}\right)^{2}+\left(r-\frac{1}{T}\right) \frac{\partial G}{\partial t}-\frac{r}{T}=0 .
$$

We introduce the Hamiltonian $H(p)$ and the generalized momentum $p$,

$$
H(p)=-\frac{\partial G}{\partial t}, \quad p=\frac{\partial G}{\partial x},
$$

then we obtain the equation for the Hamiltonian $H(p)$ :

$$
\begin{aligned}
H^{2}-v^{2} p^{2}-r H= & \frac{1}{2}(-H-v p+r) \hat{K}(H-v p) \\
& +\frac{1}{2}(-H+v p+r) \hat{K}(H+v p) .
\end{aligned}
$$

This equation allows us to determine the front speed $V$ by the formula

$$
V=\min _{p>0}\left(\frac{H(p)}{p}\right) .
$$

For the Markovian case involving exponential running time distribution we obtain

$$
H^{2}-v^{2} p^{2}-r H+\frac{H-r}{T}=0
$$

By using this formula, we find the front speed

$$
V=\min _{p>0}\left(\frac{H(p)}{p}\right)=\frac{v \sqrt{4 r T}}{1+r T}
$$

and momentum $p^{*}$ at which the speed $V$ takes the minimum value,

$$
p^{*}=\frac{2 r}{(1-r T) V} \text {. }
$$

When $r T<1$ the front speed $V$ is less than the speed of particles $v$. When $r T \rightarrow 1$, the front speed $V \rightarrow v$ and $p^{*} \rightarrow \infty$. However, for the strong anomalous case when $\hat{K}(H \pm v p)=\tau_{0}^{-\mu}(H \pm v p)^{1-\mu}$ we obtain

$$
\begin{gathered}
H^{2}-v^{2} p^{2}-r H+\frac{1}{2 \tau_{0}^{\mu}}(H+v p-r)(H-v p)^{1-\mu} \\
+\frac{1}{2 \tau_{0}^{\mu}}(H-v p-r)(H+v p)^{1-\mu}=0
\end{gathered}
$$

It follows from (47) that $\min _{p>0}(H(p) / p)=v$ and therefore the reaction front propagates with the same speed $v$ as the Lévy walker moves: $V=v$. We believe that this is a very important result for the theory of proliferating swarming bacteria performing Lévy walk and superdiffusive cancer cells [36].

Summary. We have derived the integrodifferential wave equation for the probability density function of a position of a Lévy walker with continuous sample paths. This mesoscopic equation involves a classical wave operator together with memory integrals describing a spatiotemporal coupling of Lévy walk. It is valid at all times and it does not involve any long-time large-scale approximations. It generalizes the well-known telegraph equation obtained from the persistent random walk with the exponential running time distribution. We have also implemented the nonlinear reaction into a Lévy transport and derived a reaction-transport equation that generalizes the well-known reaction-telegraph equation [7]. By using Hamilton-Jacobi techniques, we have developed the theory of wave propagation in reaction-transport systems involving Lévy diffusion and KPP kinetics. Our approach is particularly helpful because it is difficult to take into account nonlinear terms within the standard approach involving integral equations (see the similar problems for subdiffusion [37]). In a similar way one can take into account the nonlinear Lévy particles interactions [38] and Lévy walk with nonlinear death process [21]. Our theory can be useful to formulate the impact of the external force or the chemotactic substance on the random movement of particles with finite velocities.

Acknowledgments. This work was funded by EPSRC Grant No. EP/J019526/1 "Anomalous reaction-transport equations." The author gratefully acknowledges the hospitality of the Isaac Newton Institute, Cambridge and thanks N. Korabel and V. Mendez for very useful discussions during the program Coupling Geometric PDEs with Physics for Cell Morphology, Motility and Pattern Formation. The author thanks E. Barkai for useful suggestions.
[1] J. Klafter and I. M. Sokolov, First Steps in Random Walks. From Tools to Applications (Oxford University Press, Oxford, 2011).

[2] P. Barthelemy, J. Bertolotti, and D. S. Wiersma, Nature (London) 453, 495 (2008).

[3] D. A. Kessler and E. Barkai, Phys. Rev. Lett. 108, 230602 (2012).

[4] T. H. Harris et al., Nature (London) 486, 545 (2012).

[5] K. Chen, B. Wang, and S. Granick, Nat. Mater. 14, 589 (2015).
[6] G. M. Viswanathan, S. V. Buldyrev, S. Havlin, M. G. da Luz, E. P. Raposo, and H. E. Stanley, Nature (London) 401, 911 (1999); N. E. Humphries et al., ibid. 465, 1066 (2010); M. de Jager, F. J. Weissing, P. M. J. Herman, B. A. Nolet, and J. van de Koppel, Science 332, 1551 (2011).

[7] V. Mendez, D. Campos, and F. Bartumeus, Stochastic Foundations in Movement Ecology (Springer Science \& Business Media, New York, 2013). 
[8] V. Zaburdaev, S. Denisov, and J. Klafter, Rev. Mod. Phys. 87, 483 (2015).

[9] J. Klafter, A. Blumen, and M. F. Shlesinger, Phys. Rev. A 35, 3081 (1987); A. Blumen, G. Zumofen, and J. Klafter, ibid. 40, 3964 (1989).

[10] V. Yu. Zaburdaev and K. V. Chukbar, JETP 94, 252 (2002).

[11] A. Dhar, K. Saito, and B. Derrida, Phys. Rev. E 87, 010103(R) (2013).

[12] I. M. Sokolov and R. Metzler, Phys. Rev. E 67, 010101(R) (2003).

[13] R. Metzler, Phys. Rev. E 62, 6233 (2000).

[14] E. Barkai and R. Silbey, J. Phys. Chem. B 104, 3866 (2000).

[15] R. Friedrich, F. Jenko, A. Baule, and S. Eule, Phys. Rev. Lett. 96, 230601 (2006).

[16] V. M. Kenkre, E. W. Montroll, and M. F. Shlesinger, J. Stat. Phys. 9, 45 (1973).

[17] V. Mendez, S. Fedotov, and W. Horsthemke, Reaction-Transport Systems (Springer, Berlin, 2010).

[18] D. R. Cox and H. D. Miller, The Theory of Stochastic Processes (Methuen, London, 1965).

[19] M. O. Vlad, V. T. Popa, and E. Segal, Phys. Lett. A 100, 387 (1984); M. O. Vlad and J. Ross, Phys. Rev. E 66, 061908 (2002).

[20] R. Ferrari, A. J. Manfroi, and W. R. Young, Physica D 154, 111 (2001).

[21] S. Fedotov, A. Tan, and A. Zubarev, Phys. Rev. E 91, 042124 (2015).

[22] J. Masoliver, K. Lindenberg, and G. H. Weiss, Physica A 157, 891 (1989).

[23] G. Zumofen and J. Klafter, Phys. Rev. E 47, 851 (1993).

[24] M. Kac, Rocky Mountain J. Math. 4, 497 (1974).
[25] E. Korobkova et al., Nature (London) 428, 574 (2004).

[26] A. Di Crescenzo, Adv. Appl. Probab. 33, 690 (2001); A. Di Crescenzo and B. Martinucci, in Computer Aided Systems Theory-EUROCAST 2007, Lecture Notes in Computer Science Vol. 4739 (Springer, Berlin, 2007), pp. 163-170.

[27] E. Scalas, R. Gorenflo, F. Mainardi, and M. Raberto, Fractals 11, 281 (2003).

[28] S. Fedotov and S. Falconer, Phys. Rev. E 87, 052139 (2013).

[29] M. M. Meerschaert, D. A. Benson, H. P. Scheffler, and P. BeckerKern, Phys. Rev. E 66, 060102 (2002).

[30] B. Baeumer, M. M. Meerschaert, and J. Mortensen, Proc. Am. Math. Soc. 133, 2273 (2005).

[31] P. Becker-Kern, M. M. Meerschaert, and H. P. Scheffler, Ann. Probab. 32, 730 (2004).

[32] V. V. Uchaikin and R. T. Sibatov, J. Phys. A 44, 145501 (2011).

[33] M. Magdziarz, H. P. Scheffler, P. Straka, and P. Zebrowski, Stoch. Proc. Appl. 125, 4021 (2015).

[34] D. Froemberg, M. Schmiedeberg, E. Barkai, and V. Zaburdaev, Phys. Rev. E 91, 022131 (2015).

[35] A. Rebenshtok, S. Denisov, P. Hanggi, and E. Barkai, Phys. Rev. Lett. 112, 110601 (2014).

[36] G. Ariel, A. Rabani, S. Benisty, J. D. Partridge, R. M. Harshey, and A. Be'er, Nat. Commun. 6, 8396 (2015); C. T. Mierke, New J. Phys. 15, 015003 (2013).

[37] S. Fedotov, Phys. Rev. E 88, 032104 (2013); P. Straka and S. Fedotov, J. Theor. Biol. 366, 71 (2015).

[38] M. de Jager, F. Bartumeus, A. Koelzsch, F. J. Weissing, G. M. Hengeveld, B. A. Nolet, P. M. J. Herman, and J. van de Koppel, Proc. R. Soc. B 281, 20132605 (2014). 